\title{
Effect of a leisure activity such as talking on the blood glucose response in healthy subjects after a carbohydrates load
}

\author{
Y. Farooqi and M. Chopra \\ School of Pharmacy and Biomedical Science, University of Portsmouth, Portsmouth PO1 2DT
}

Several factors can influence the glycaemic response of an individual to a carbohydrate meal. These factors include the glycaemic index (GI) of foods, co-ingestion of other macronutrients such as protein, fibre as well as mental and physical activity. ${ }^{(1)}$ The effect of talking behaviour on glycaemic response of individuals has not been reported before. In the present study we have examined the effect of quiet and talking behaviour on the glycaemic response of healthy volunteers following a glucose load. Both high (lucozade) and medium (muffins) GI foods were tested.

Ten healthy subjects (five male, five female) age 20-25 years participated in the study. Baseline glucose measurements were done in the morning after an overnight fast of at least $9 \mathrm{~h}$. Subjects were asked to consume $44 \mathrm{~g}$ of carbohydrate from $258 \mathrm{ml}$ lucozade or from chocolate chip muffins and their blood glucose levels of their pin prick samples were measured with glucose meter every 20 min for up to $2 \mathrm{~h}$. Subjects were asked to remain quiet or maintain a loud behaviour during the experiment and the effect on blood glucose changes was monitored. Each subject acted as its own control that is, repeated the experiment four times and the glucose response was measured with high and medium GI glucose load in the presence and absence of quiet behaviour. The blood glucose changes are shown in the following table.

Table. Mean (SD) values for blood glucose (mmol/l) after the consumption of high GI (Lucozade) and medium GI (muffin) foods following a loud and quiet behaviour

\begin{tabular}{|c|c|c|c|c|c|c|c|}
\hline & Baseline & $20 \mathrm{~min}$ & $40 \mathrm{~min}$ & $60 \mathrm{~min}$ & $80 \mathrm{~min}$ & $100 \min$ & $120 \mathrm{~min}$ \\
\hline Lucozade (talking) & $4.66(0.4)$ & $7.09(0.8)$ & $7.99(1.1)$ & $6.48(0.7)$ & $6.37(0.6)$ & $5.54(0.5)$ & $4.34(0.4)$ \\
\hline Lucozade (quiet) & $4.62(0.4)$ & $7.30(0.6)$ & $8.42(1.3)$ & $7.31(1.7)$ & $6.77(1.1)$ & $6.11(0.6)$ & $5.78 * *(0.4)$ \\
\hline Muffins (talking) & $4.56(0.4)$ & $5.77(0.5)$ & $6.18(0.5)$ & $5.67(0.6)$ & $5.27(0.4)$ & $4.93(0.6)$ & $4.40(0.4)$ \\
\hline Muffins (quiet) & $4.79(0.6)$ & $5.84(0.5)$ & $6.00(0.6)$ & $5.70(0.6)$ & $5.87 *(0.5)$ & $5.54 * *(0.3)$ & $5.35^{* *}(0.3)$ \\
\hline
\end{tabular}

Comparison of loud and quiet behaviour on blood glucose response with lucozade and muffin (Wilcoxon Rank Sum test). $* P<0.05$ and $* * P<0.01$.

The results show that talking compared with a quiet behaviour can influence the blood glucose response after a carbohydrate load. This therefore could influence the results of the studies that are conducted to examine the blood glucose response after a carbohydrate meal. Also, a similar experiment as above in type 2 diabetic patients may inform whether these patients can benefit from their lifestyle behaviour in response to a carbohydrate food.

1. Foster-Powell K, Holt SH \& Brand-Miller JC (2002) Am J Clin Nutr 76, 5-56. 